\title{
La investigación sobre XML legislativo en España
}

\author{
M. ${ }^{a}$ Mercedes Martínez González \\ Dámaso-Javier Vicente Blanco \\ Universidad de Valladolid (España)
}

\section{Resumen}

Se presentan los artículos del número 1 de volumen 15 de la revista Scire: Representación y Organización del Conocimiento (en.-jun. 2009), que recoge una selección revisada de los trabajos presentados en el I Simposio sobre XML Legislativo en España.

Palabras clave: Representación del conocimiento. Organización del conocimiento. Información jurídica. Legislación. XML. Web semántica.

\section{Abstract}

The articles published in the number 1 of the volume 15 of Scire: Representación y Organización del Conocimiento (Jan.-Jun. 2009) are presented, which consist of a reviewed selection of the papers presented at the I Spanish Symposium on Legislative XML.

Keywords: Knowledge representation. Knowledge organization. Legal information. Legislation. XML. Semantic web.

El presente volumen de la revista Scire recoge una selección de los trabajos presentados en el I Simposio sobre XML Legislativo en España, celebrado en la Facultad de Derecho de la Universidad de Valladolid los días 15 y 16 de noviembre de 2007, donde se dio cuenta de las experiencias españolas en la aplicación de la tecnología XML a la manipulación electrónica de textos legislativos.

El lenguaje XML ha demostrado ser el soporte más adecuado para la provisión de estándares para los documentos legislativos en formato digital. Las cualidades de los documentos legislativos (estructura, articulación, comparabilidad, interrelación, referenciación, etcétera), así como las necesidades de manejo por los usuarios, hacen de la tecnología XML una herramienta apropiada. En ello inciden especialmente las posibilidades de este lenguaje para el tratamiento de la información recogida en los textos jurídicos, ofreciendo información sobre esa información (metainformación), sobre su aptitud para el reconocimiento de tal información y, en resumen, sobre su indudable utilidad, aplicada, junto con tecnologías de búsqueda y recuperación de información, a tales fines.

Scire. $15: 1$ (en.-jun. 2009) 9-13. ISSN 1135-3716. 
Una prueba de esta adecuación está en la multiplicidad de proyectos existentes en Europa (MetaLex, NormaInRete, etcétera), en la adopción de este lenguaje XML por la Oficina de Publicaciones de la Comisión Europea como soporte para la legislación comunitaria o en su consolidación en las editoriales comerciales de todo el mundo que suministran textos legislativos. España no es una excepción a esta tendencia. El Senado, varias administraciones públicas, iniciativas privadas, y grupos de investigación trabajan para ofrecer y mejorar progresivamente el acceso a la legislación en formato electrónico, tanto para profesionales como para ciudadanos, mediante el uso de XML.

En consecuencia, la potencialidad y la relevancia práctica del lenguaje XML en el tratamiento de la información jurídica hacen que la investigación y la industria que tienen por objeto el llamado XML legislativo (es decir, el uso del lenguaje XML para el tratamiento de textos jurídicos) merezcan una singular atención, así como su uso práctico por los operadores jurídicos. Y esta atención debe tomar en consideración el hecho real de que en la materia se concitan intereses multidisciplinares, tanto desde la perspectiva jurídica como desde el punto de vista de la ingeniería informática o desde el de la biblioteconomía y la documentación.

En 2006, los coordinadores de este monográfico fuimos invitados a presentar una comunicación sobre el estado del XML legislativo en España (Spain on going legislative XML projects) en el V Legislative XML Workshop, celebrado en Fiesole (Florencia) del 14 al 16 de junio de ese año. Allí coincidimos con miembros de equipos de trabajo españoles en la materia, a los que pertenecen algunas de las contribuciones que aparecen en este número, y consideramos la conveniencia de congregar en una reunión científica a los investigadores y profesionales vinculados en España con el desarrollo del XML legislativo.

El I Simposio sobre XML Legislativo en España nació con vocación de iniciar un foro de discusión donde la comunidad española, entidades públicas, empresas e investigadores puedan compartir sus experiencias, divulgarlas y discutir sobre la evolución futura en España del lenguaje XML como lenguaje de los textos jurídicos. Con su puesta en marcha se ha perseguido iniciar un proceso de interrelación e intercambio multidisciplinar de experiencias entre los investigadores que se dedican en España a los problemas del tratamiento electrónico de textos jurídicos a través de la tecnología XML (ingenieros informáticos, documentalistas y juristas) y evaluar los resultados alcanzados hasta la fecha.

Entre los objetivos del Simposio que expresamente nos planteamos se encontraban los de enmarcar la materia en el contexto del gobierno electrónico, establecer una relación de diálogo entre los investigadores en la materia y las empresas que desarrollan aplicaciones en el campo y fomentar el desarrollo en España de la investigación sobre la aplicación de la tecnología XML a los textos jurídicos, así como difundir su importancia en el ámbito jurídico y documental. El Simpo- 
sio se dirigió a profesionales (juristas, documentalistas, técnicos) interesados en el tema de XML legislativo, investigadores y estudiantes interesados en conocer las posibilidades de este campo. Los temas que entendíamos podían ser tratados se encuadraban en cinco áreas de interés: $a$ ) la investigación informática en España sobre XML legislativo: experiencias y resultados; $b$ ) la investigación documentalista en España sobre XML legislativo: experiencias y resultados; $c$ ) los juristas y la aplicación de la tecnología XML a los textos normativos; d) la técnica legislativa y el tratamiento informático de textos jurídicos; $e$ ) el lenguaje XML aplicado a los textos legislativos y el gobierno electrónico; $f$ ) las experiencias empresariales y las de las instituciones públicas en la utilización de XML con los textos jurídicos.

Los estudios aquí reunidos recogen nueve de las ponencias y comunicaciones con las que contó el Simposio. Todos ellos han sido sometidos a un proceso de estudio de la calidad a través de un procedimiento de revisión anónima por pares (peer review). Por vez primera se hace una recopilación de las experiencias españolas comerciales, institucionales y de investigación sobre el XML legislativo. Estamos ante un conjunto de trabajos y estudios que son y están entre los fundamentales que se han llevado a cabo en España sobre la materia, bien entendido que hemos considerado el XML legislativo en su sentido estricto, es decir, restringiéndolo exclusivamente al uso del lenguaje XML para el tratamiento de textos jurídicos. Otros supuestos próximos o relacionados de indudable interés, como los relativos a las ontologías, no están presentes, por cuanto no tienen, sensu stricto, el carácter de XML legislativo.

El primer grupo de trabajos cumple la función de plantear el marco en el que se insertan los estudios concretos sobre las experiencias españolas relativas al XML legislativo. El texto que abre este grupo lleva por título «EUR-Lex: el sistema de información jurídica de la Unión Europea» y corresponde a la exposición de José Antonio Domínguez Rojas, de la Sección de Trabajos Documentales de la Unidad EUR-Lex de la Oficina de Publicaciones de las Comunidades Europeas. Al ser una realidad europea, la experiencia del sistema EUR-Lex aparece como un paradigma en el tratamiento informático de textos jurídicos, conocido y con una autoridad imposible de ignorar sobre la realidad española. El segundo texto corresponde al estudio de M. ${ }^{a}$ Luisa Alvite Díez, profesora de Biblioteconomía y Documentación de la Universidad de León, que lleva a cabo una detallada exposición sobre «Las bases de datos jurídicas y el uso del lenguaje XML en España» realizando un recorrido por las experiencias españolas en la materia. El estudio que lleva por título «Perspectivas sobre el uso de la web semántica en el tratamiento de información y documentación legislativa», de Francisco Javier García Marco, profesor de Biblioteconomía y Documentación de la Universidad de Zaragoza, ocupa el tercer lugar y permite enmarcar los trabajos de XML legislativo en el contexto de la web semántica. Finalmente, cerrando este primer grupo de trabajos, se encuentra el de Scire. $15: 1$ (en.-jun. 2009) 9-13. ISSN 1135-3716. 
María José Vañó Vañó, profesora de Derecho Mercantil de la Universidad de Valencia, que trata sobre «Integración de la documentación legal electrónica a través de LEXML».

El segundo grupo recoge dos trabajos en los que se muestran sendas experiencias de uso del lenguaje XML legislativo, una institucional y otra de carácter comercial. Se trata de dos experiencias bien consolidadas, ya con una trayectoria sólida en su funcionamiento, y muy representativas de lo que se hace en nuestro país en la materia. La de las instituciones públicas está representada por Armando Alonso Peri, del Principado de Asturias, y lleva por título «SIDRA: XML en la gestión y explotación de la documentación legislativa», mientras que la de las empresas comerciales la expone, a través del trabajo «La gestión documental en Lex Nova: producción y publicación», Leandro Escudero, de la editorial Lex Nova.

El tercer y último grupo de trabajos corresponde a las experiencias de investigación. En él se recogen tres experiencias que entendemos tienen carácter significativo. La primera, cuyos autores son y Javier de Andrés Rivero y Rafael Hernández Marín, de la Universidad de Murcia, lleva por título «CRONOLEX: sistema para la representación dinámica de cuerpos legales». La segunda experiencia se presenta en el trabajo de M. ${ }^{a}$ del Carmen Arellano Pardo y J. Tomás Nogales Flores, de la Universidad Carlos III de Madrid, que lleva por título «Lenguajes de marcado y documentación jurídica: experiencias del grupo TecnoDoc de la UC3M». Finalmente, la tercera lleva por título «Estructura, semántica, extracción de información y XML legislativo: experiencias en la Universidad de Valladolid» y son sus autores el grupo de la Universidad de Valladolid formado por M. ${ }^{a}$ Mercedes Martínez González, Dámaso-Javier Vicente Blanco, Pablo de la Fuente Redondo, Joaquín Adiego Rodríguez, Alma María Pisabarro Marrón y José Manuel Sánchez Felipe. Todos ellos plantean esquemas que permiten modelar textos jurídicos en formato XML e indagan sobre distintos aspectos de su manipulación, como la consolidación, la navegación y explotación de relaciones o los mejores vocabularios de marcado.

Desde esa perspectiva, esta publicación y el Simposio de Valladolid han conseguido el objetivo fundamental planteado, al reunir y mostrar públicamente los trabajos de la comunidad que trabaja en España sobre la aplicación de la tecnología XML a la información legislativa (investigadores, empresas e instituciones). Si gracias al evento fue posible conocer e intercambiar experiencias, cuando antes solamente teníamos referencias unos de otros, la publicación permite ofrecer esas experiencias a la comunidad científica. La voluntad es que este sea el inicio de un foro de discusión sobre la materia que pueda continuar en el futuro. En consecuencia, el debate no concluye aquí. La página web del Simposio (donde se pueden encontrar el programa y los PDF de las presentaciones) sigue viva como instrumento de comunicación (http://www.infor.uva.es/xmleg/), y existe el compromiso de realizar sucesivas ediciones del Simposio en el futuro.

Scire. $15: 1$ (en.-jun. 2009) 9-13. ISSN 1135-3716. 
Como parece lógico en un campo tan específico como el que nos ocupa, la comunidad que trabaja en él en España es reducida. Ello no es ningún impedimento para que, tal y como muestran los trabajos que se reúnen en esta publicación, la experiencia española en el XML legislativo sea sólida, tanto en el campo de la investigación como en los desarrollos en funcionamiento en los ámbitos empresarial e institucional. Ello no obsta para que se sea consciente de que es necesario seguir trabajando y colaborando para avanzar en aspectos como la interoperabilidad (que facilite aún más el intercambio de datos e información), la recuperación de información y la explotación de la semántica en los sistemas de información jurídicos.

\section{Referencias}

Alonso Peri, Armando (2009). SIDRA: XML en la gestión y explotación de la documentación jurídica. // Scire: Representación y Organización del Conocimiento. 15:1 (en.jun. 2009) 111-124.

Alvite Díez, M. ${ }^{a}$ Luisa (2009). Las bases de datos jurídicas y el uso del lenguaje XML en España. // Scire: Representación y Organización del Conocimiento. 15:1 (en.-jun. 2009) 33-57.

Andrés Rivero, Javier de; Hernández Marín, Rafael (2009). CRONOLEX: sistema para la representación dinámica de cuerpos legales. // Scire: Representación y Organización del Conocimiento. 15:1 (en.-jun. 2009) 133-147.

Arellano Pardo, M. ${ }^{a}$ del Carmen; Nogales Flores, J. Tomás (2009). Lenguajes de marcado y documentación jurídica: experiencias del grupo TecnoDoc de la UC3M. // Scire: Representación y Organización del Conocimiento. 15:1 (en.-jun. 2009) 149-171.

Domínguez Rojas, José Antonio (2009). EUR-Lex: el sistema de información jurídica de la Unión Europea. // Scire: Representación y Organización del Conocimiento. 15:1 (en.-jun. 2009) 15-32.

Escudero Atienza Leandro (2009). La gestión documental en Lex Nova: producción y publicación. // Scire: Representación y Organización del Conocimiento. 15:1 (en.-jun. 2009) 125-131.

García Marco, Francisco Javier (2009). Perspectivas sobre el uso de la Web Semántica en el tratamiento de información y documentación legislativa. // Scire: Representación y Organización del Conocimiento. 15:1 (en.-jun. 2009) 59-85.

Martínez González, M. ${ }^{a}$ Mercedes; Vicente Blanco, Dámaso-Javier; Fuente Redondo, Pablo de la; Adiego Rodríguez, Joaquín; Pisabarro Marrón, Alma María; Sánchez Felipe, José Manuel (2009). Estructura, semántica, extracción de información y XML legislativo: experiencias en la Universidad de Valladolid. // Scire: Representación y Organización del Conocimiento. 15:1 (en.-jun. 2009) 173-186.

Vañó Vañó, María José (2009). Integración de la documentación legal electrónica a través de LEXML. // Scire: Representación y Organización del Conocimiento. 15:1 (en.-jun. 2009) $87-110$.

Scire. $15: 1$ (en.-jun. 2009) 9-13. ISSN 1135-3716. 